\title{
La lógica de los hábitos prescriptivos de los psiquiatras
}

\author{
Soroya Mayet Senior House Officers \\ Michelle Smith Senior House Officers \\ Dele Olajide Consultant Psychiatrist \\ South London and Maudsley NHS Trust, \\ Maudsley Hospital, London, SE5 8AZ \\ UNITED KINGDOM
}

\begin{abstract}
RESUMEN - Objetivo y Método: Este estudio examina las razones que subyacen a la prescripción farmacológica en psiquiatría. Se pidió a varios psiquiatras de diferentes niveles clínicos de un dispositivo de salud mental que completaran una encuesta que examinaba especialmente sus selecciones de fármacos y el uso de polifarmacia en pacientes diagnosticados de esquizofrenia, manía y depresión.

Resultados: entre los que respondieron, se encontró que la "experiencia personal" era la razón más importante al prescribir. Entre los pre-residentes ("Senior House Officers", SHO) era significativamente más probable que recetaran como resultado de la "influencia de sus colegas".

Implicaciones Clínicas: Aunque los hábitos prescriptivos se atribuían a la "experiencia personal", la elección del medicamento y el uso de polifarmacia, eran acordes con la evidencia disponible.
\end{abstract}

\section{Introducción}

La prescripción farmacológica es compleja y su práctica no siempre es adecuada (Taylor 2000a). Se han demostrado inconsistencias al recetar en todas las especialidades médicas (Hogerzeil 1995), incluída la Psiquiatría (Taylor 1998).

Se piensa que son numerosos los factores que influyen en los hábitos prescriptivos de los médicos. Dickinson-Mulinga (1998) en- contró que la evidencia personal era un factor predominante que subyacía al por qué los psiquiatras recetan un fármaco en particular. Nuestro estudio examina si la base fundamental de los hábitos prescriptivos de los psiquiatras se ha alterado con el énfasis creciente en las prácticas basadas en datos ("evidencias") y en las guías de actuación clínicas.

Analizando las razones que gobiernan los hábitos prescriptivos de los psiquiatras se 
pueden mejorar los patrones de prescripción y la efectividad clínica.

\section{Objetivos}

Este trabajo analiza las razones que subyacen a la prescripción en psiquiatría, examinando especialmente la elección de medicamento por parte de los psiquiatras y el uso de polifarmacia en la esquizofrenia, la depresión y la manía.

\section{Método}

Se invitó a participar en este estudio a los psiquiatras de los hospitales Maudsley y Bethlem Royal durante el período de enero a marzo de 2002. Se les envió un cuestionario semi-estructurado de tres preguntas a todos los doctores con los que se había contactado, bien personalmente, a través sus secretarias, o bien por correo postal o electrónico.

La primera pregunta indagaba acerca de la elección del medicamento por parte del prescriptor en un primer episodio de psicosis esquizofrénica, depresión y manía aguda no complicadas. La segunda pregunta determinaba si el prescriptor se engranaba en el uso de polifarmacia (administración concomitante de 2 ó más fármacos) en dichos trastornos. Esta pregunta examinaba el uso de antipsicó- ticos combinados con anticolinérgicos, así como la combinación de varios antipsicóticos en la esquizofrenia. Además, se evaluó la acción combinada de estabilizadores del humor y antipsicóticos en la manía o estabilizadores del humor y antidepresivos en la depresión.

La tercera pregunta indagaba sobre las razones del prescriptor para decidir el medicamento de primera elección y el uso de polifarmacia para cada uno de los trastornos. Las posibles respuestas fueron: experiencia personal, medicina basada en la evidencia, consideraciones económicas, influencia de las empresas farmacéuticas, influencia de colegas, preferencias del paciente y la guía de actuación de prescripción del Maudsley.

Los datos fueron recogidos y analizados utilizando el programa estadístico SPSS-10.

\section{Resultados}

De 150 cuestionarios enviados, 104 fueron devueltos, obteniendo un porcentaje de respuesta del $69 \%$. El 33,7\% eran consultores, un $17,8 \%$ residentes especialistas ("specialist registrars") y un 48,5\% pre-residentes (Senior House Officers, SHO). El 54\% de los encuestados eran mujeres y el $46 \%$ eran varones, con una media de 9,5 años desde la licenciatura. La etnia de los encuestados, de acuerdo con la clasificación de etnicidad OPCS, fue $72 \%$ caucasianos, $14 \%$ hindúes,

Tabla I

Orden de los fármacos de primera elección para esquizofrenia, depresión y manía

\begin{tabular}{lclclc} 
Esquizofrenia & $\%$ & Depresión & $\%$ & Manía & $\%$ \\
\hline Olanzapina & 55,2 & Fluoxetina & 35,6 & Litio & 39,1 \\
Risperidona & 37,5 & Paroxetina & 27,6 & Antipsicóticos & 29,9 \\
Quetiapina & 6,3 & Citalopram & 21,8 & Valproato Sódico & 24,1 \\
Amisulpirida & 1 & Venlafaxina & 5,8 & Benzodiacepina & 4,6 \\
& & Tricíclicos & 2,3 & Carbamacepina & 2,3 \\
& & Otros & 6,9 & & \\
\hline
\end{tabular}


$6 \%$ negros africanos, $1 \%$ negros caribeños, $3 \%$ chinos y $3 \%$ otras etnias.

La Tabla I muestra el orden del medicamento de primera elección en el primer episodio de psicosis esquizofrénica, depresión y manía aguda no complicadas. Un 34\% de los encuestados reconocieron la prescripción de anticolinérgicos con antipsicóticos, y se utilizó combinación de antipsicóticos en un $33 \%$. Un $96 \%$ declararon que utilizaban un tratamiento combinado de estabiliza- dores del humor con antipsicóticos en manía y un $70 \%$ utilizaban una combinación de estabilizadores del humor y antidepresivos en la depresión.

Las razones para la elección del fármaco en la esquizofrenia (Figura 1), depresión y manía fueron "experiencia personal", seguido de "medicina basada en datos" ("evidencias") y después "influencia de los colegas" y guía de actuación para la prescripción del Maudsley". Sin embargo, las razones para la

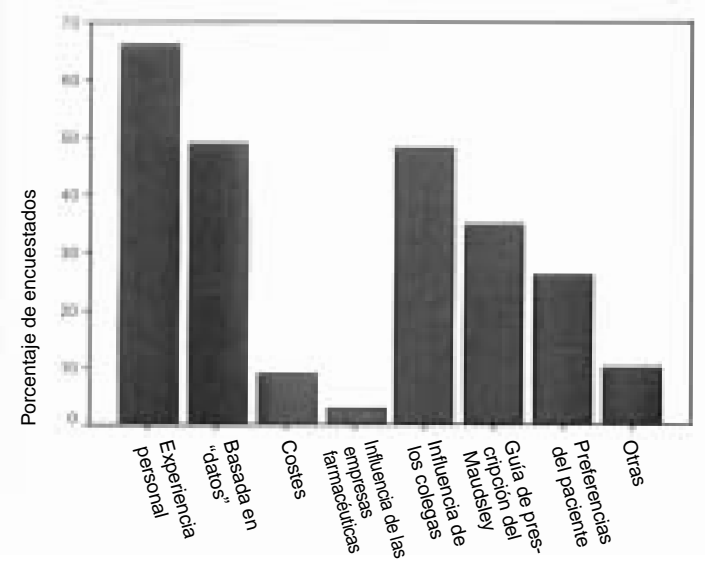

Figura 1. Razones para la elección de fármacos en la esquizofrenia.

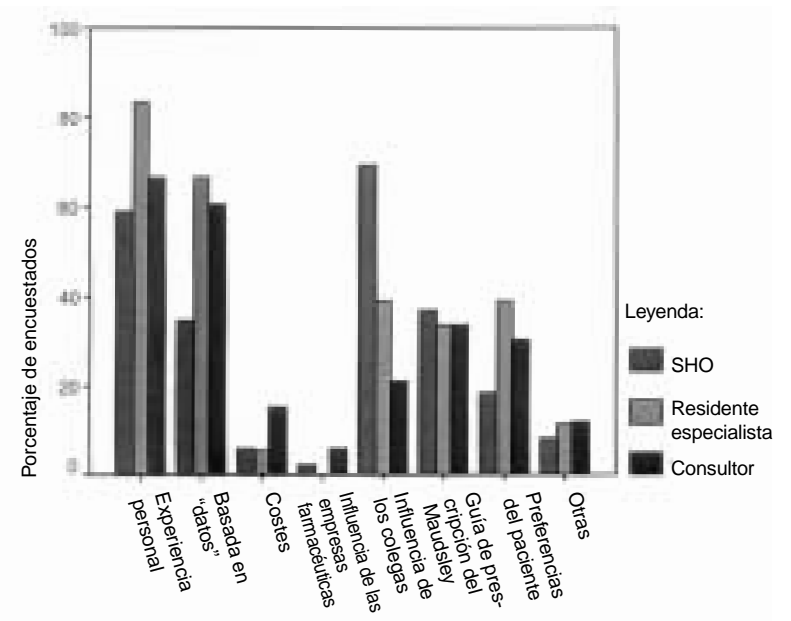

Figura 2. Razones para la elección de fármacos en la esquizofrenia, por niveles de los clínicos. 
combinación de fármacos fueron "experiencia personal", seguida de "influencia de los colegas" y, por último, la "medicina basada en datos" ("evidencias").

Los SHO eran más proclives a citar la "influencia de los colegas" que sus colegas senior $(\mathrm{p}<0,05)$. Para el tratamiento combinado en la manía, los consultores y residentes especialistas citaban más la "experiencia personal" que los SHO (Figura 2). Se halló una tendencia a que los varones respondieran con mayor frecuencia que sus prescripciones eran "basadas en datos" ("evidencias").

\section{Discusión}

Este estudio se centra en los hábitos prescriptivos de los psiquiatras y en las razones de sus elecciones. Los consultores y los residentes especialistas atribuyeron sus hábitos prescriptivos a la "experiencia personal". Nuestro hallazgo es consistente con el estudio de Dickinson-Mullinga en 1998. En comparación con sus colegas senior, los SHO atribuyeron sus hábitos prescriptivos a la "influencia de los colegas". Esto no es sorprendente, ya que los SHO es más probable que dependan de la supervisión de sus colegas senior por su mayor pericia. La atribución de los consultores de sus hábitos prescriptivos a la "experiencia personal" puede ser un reflejo de su familiarización con la bibliografía y su confianza basada en varios años de experiencia. Nuestro estudio muestra que la "influencia de las compañías farmacéuticas" fue el factor menos importante en los hábitos prescriptivos. "Preferencias del paciente" y "costes" fueron sorprendentemente poco citados, a pesar del creciente énfasis de los gobiernos hacia el cuidado centrado en el paciente y la relación coste-efectividad (National Institute of Clinical Excellence 2002).
La olanzapina fue claramente preferida como el fármaco de primera elección en el primer episodio de psicosis esquizofrénica. Esto es consistente con los hallazgos previos (Taylor 1998b). Quizás la “experiencia personal" era más importante que "basarse en datos" debido a la evidencia contradictoria para la prescripción de antipsicóticos atípicos (Gedes 2000, Mir 1998). Las “consideraciones económicas" no fueron estimadas como un factor importante a la hora de elegir antipsicóticos atípicos; este dato es consistente con el hecho de que la olanzapina fuera preferida a la risperidona, a pesar del mayor coste unitario de la primera. El uso de risperidona y olanzapina en un $95 \%$ de los encuestados es consistente con las actuales guías de actuación, lo que sugiere que la "experiencia personal" puede no ser tan subjetiva como se pensaba previamente.

La combinación de antipsicóticos era defendida por un $33 \%$ de los encuestados, hallazgo similar a un estudio previo (Taylor 2002). Existen pocos datos a favor de la combinación de antipsicóticos y, en cambio, hay datos que sugieren una mayor mortalidad con esta práctica (Waddingrton 1998). La combinación de antipsicóticos debería ser evitada cuando fuera posible, excepto en el "aumento" con clozapina (Taylor 2002). Esta combinación puede sugerir que la razón de la "experiencia personal" no estaba basada en datos y sí bastante en la experiencia subjetiva del clínico. Aproximadamente un tercio de nuestra muestra utilizaba una combinación de anticolinérgicos con antipsicóticos. Esta práctica está solamente recomendada como tratamiento a corto plazo del parkinsonismo inducido por neurolépticos (Stanill 1998). El uso crónico de medicación anticolinérgica puede no sólamente conducir a un déficit cognoscitivo, sino que también puede ser utilizado como un euforizante por los pacientes. 
Se comprobó que el litio era el medicamento de primera elección en la manía aguda, lo cual está en la línea de las actuales guías de actuación (Taylor 2001, APA 1994). Por lo tanto, la "experiencia personal" en el tratamiento de la manía aguda puede estar basada ampliamente en las guías de actuación al uso, antes que estar basada en datos (Soraes 1997). Se comprobó que los antipsicóticos eran la siguiente elección más frecuente en el tratamiento de la manía aguda, una práctica que es consistente con la bibliografía sobre el tratamiento de la manía (Soares 1997). Casi todos los encuestados utilizaban una combinación de estabilizadores del humor y un antipsicótico. Esta práctica puede indicar que una alta proporción de casos de manía responden a una combinación de antipsicóticos y estabilizadores del humor.

Nuestros encuestados indicaron que su primera elección de tratamiento farmacológico en el primer episodio de depresión fue fluoxetina. Aunque no hay diferencia significativa, fármacológicamente hablando, en la efectividad clínica entre los diferentes antidepresivos, los inhibidores selectivos de la recaptación de la serotonina son mejor tolerados que los antidepresivos tricíclicos (Clinical Evidence 2000). Recientemente está disponible la versión genérica y más barata de la fluoxetina desde que a "Prozac" se le terminó la patente (BNF 2002). De esta manera, los datos y las consideraciones acerca del coste pueden influir en la idea de "experiencia personal" de los prescriptores. La combinación de un estabilizador del humor y un antidepresivo era utilizada por más del 60\% de los encuestados. Ésta es una opción de tratamiento establecida para la depresión refractaria (Bauer 1999).

Si bien nosotros hemos documentado que la "experiencia personal" es la razón más importantre para recetar medicamentos entre nuestros encuestados, este hecho puede, en realidad, reflejar un cuerpo de conocimientos adquiridos basados en la bibliografía cientifica puesto que, en esta muestra, la "experiencia personal" es coherente con las "prácticas basadas en datos". El presente estudio no establece lo que los prescriptores quieren decir con "experiencia personal", pero sus elecciones de medicamentos son acordes con los datos disponibles y las guías de actuación, excepto en el caso de la combinación de antipsicóticos, en la que existe escasa evidencia que apoye su uso.

Una limitación de nuestro estudio es el pequeño tamaño de la muestra. Reconocemos también que el instrumento utilizado es un cuestionario de auto-informe, que puede no ser completamente fiable. Sin embargo, el estudio ha sido consistente con las recientes auditorías de prescripción en este mismo complejo hospitalario.

\section{Conclusiones}

Nuestros resultados nos han permitido recoger algunos indicadores de la calidad de la prescripción y hasta qué punto se cumplen los estándares recomendados. Este estudio ha permitido mostrar que, a pesar de la importancia dada a la "medicina basada en datos" y a las guías de actuación clínicas, los médicos continúan dependiendo de la "experiencia personal" como principal determinante de sus hábitos prescriptivos. Sin embargo, es importante reconocer que, en la mayoría de los casos, la "experiencia personal" coincide con los actuales datos disponibles y guías de actuación. La necesidad de desbancar las experiencias personales subjetivas por la "medicina basada en datos" debe permanecer como un importante objetivo que fomente una óptima prescripción. Sin embargo, debe- 
mos reconocer que cada medicamento puede afectar de manera diferente a cada individuo $y$, en esas circunstancias, el prescriptor puede utilizar su "experiencia personal" para conseguir un resultado exitoso.

\section{Bibliografía}

AMERICAN PSYCHIATRIC ASSOCIATION Practice guideline for treatment of patients with bipolar disorder. American Journal of Psychiatry, 151, 1-36, 1994.

BAUER, M., DOPFMER, S. Lithium augmentation in the treatment-resistant depression: meta-analysis of placebo controlled studies. Journal of Clinical Psychopharmacology, 19, 427-434, 1999.

BRITISH MEDICAL ASSOCIATION AND ROYAL PHARMACEUTICAL SOCIETY OF GREAT BRITAIN. 43: British National Formulary. London: British Medical Association and Royal Pharmaceutical Society of Great Britain, 2002.

Clinical Evidence 4, 522-525. British Medical Journal Publishing Group, 2000.

DICKSON-MULINGA, J. Rationale behind psychiatrists' choice of drug. Psychiatric Bulletin, 22, 677-680, 1998.

GEDDES, J., FREEMANTLE, N., HARRISON, P., BEBBINGTON, PAUL. Atypical antipsychotics in the treatment of schizophrenia: systematic overview and metaregression analysis. British Medical Journal, 321, 1371-1376, 2000 .

HOGERZEIL, H.V. Promoting rationale prescribing: an international perspective. British Journal of Clinical Pharmacology, 39, 1-6, 1995.

MIR, S., TAYLOR, D. Schizophrenia. Pharmacology Journal, 261, 55-58, 1998.

MONCRIEFF, J. Lithium: evidence reconsidered. The British Journal of Psychiatry, 171, 113-119, 1997.
NATIONAL INSTITUTE OF CLINICAL EXCELLENCE. Guidance on the use of newer (atypical) antipsychotic drugs for the treatment of schizophrenia. Technology Appraisal Guidance - 43, June, www.nice.org.uk. 2002.

SOARES, J.C., MALLINGER, A.G., GERSHON, S., The role of antipsychotic agents in the treatment of acute mania. American Journal of Psychiatry, 156, 1019-1023, 1997.

STANILL, J.K., SIMPSON, G.M. Treatment of extra pyramidal side Effects in The American Psychiatric Press textbook of Pyschopharmacology, $2^{\text {nd }}$ Edition. Eds. Schatzberg, A.F. \& Memeroff C. B., 1998.

TAYLOR, D., MIR, S., KERWIN, R. Prescribing in schizophrenia: evaluating the effect of introducing a new treatment protocol. Psychiatric Bulletin, 24, 106-108, 2000a.

TAYLOR, D., MIR, S., MACE, S. et al. A prescription survey of the use of atypical antipsychotics for hospital inpatients in the United Kingdom. International Journal of Psychiatry in Clinical Practice, 4, 41-46, 2000b.

TAYLOR, D., DRUMMOND, S., PENDLEBURY, J. Olanzapine in practice. Psychiatric Bulletin, 22, 552-554, 1998.

TAYLOR, D., McCONNELL, D., McCONNELL, H., KERWIN, R. The South London and Maudsley NHS Trust Prescribing Guidelines $6^{\text {th }}$ Ed. Martin Dunitz, London, 2001.

WADDINGTON, J.L. Mortality in schizophrenia. Antipsychotic polypharmacy and absence of adjunctive anticholinergics over the course of a 10-year prospective study. The British Journal of Psychiatry, 173, 325-9, 1998.

Dirección para correspondencia:

Dele Olajide

Consultant Psychiatrist

South London and Maudsley NHS Trust

Maudsley Hospital

London

SE5 8AZ

UNITED KINGDOM

e-mail: dele.olajide@slam-tr.nhs.uk 